\title{
Disaccharide Tripeptide Glycerol Dipalmitoyl
}

National Cancer Institute

\section{Source}

National Cancer Institute. Disaccharide Tripeptide Glycerol Dipalmitoyl. NCI Thesaurus.

Code C1575.

A lipophilic disaccharide tripeptide derivative of muramyl dipeptide (MDP) with immunomodulatory activity. Disaccharide tripeptide glycerol dipalmitoyl (DTPGDP)stimulates macrophage activity and increases serum levels of tumor necrosis factor alpha (TNF alpha), neopterin, interleukin (IL)-1 alpha, IL-1 beta, IL-6, IL-8, and IL-12, which may activate host immune system antitumor functions. DTP-GDP may be packaged in liposomes for improved delivery. The immunomodulatory effects of this agent may be superior to those of MDP. 